\title{
El papel del lenguaje en la construcción de explicaciones en la clase de ciencias en contextos bilingües a través del enfoque CLIL ${ }^{1}$
}

\section{The Role of Language in Elaborating Explanations in the Science Class in Bilingual Contexts Through the CLIL} Approach

\author{
Mauricio Mancipe Triviño \\ Cynthia Marcela Ramírez Valenzuela ${ }^{3}$
}

\begin{abstract}
Citation/ Para citar este Artículo: Mancipe, M. y Ramírez, C. (2019). El papel del lenguaje en la construcción de explicaciones en la clase de ciencias en contextos bilingües a través del enfoque CLIL. Colomb. Appl. Linguistic. J., 21(1), pp. 105-122.
\end{abstract}

Received: 3-Jun.-2018 / Accepted: 19-Mar.-2019

DOI: https://doi.org/10.14483/22487085.13095

\begin{abstract}
This paper covers the issue with respect to elaborating explanations about natural phenomena in the Science class in bilingual contexts (Spanish (L1) - English (L2)), in which the role of the language is analysed from two perspectives: communicative and explanatory. To do so, this article focuses on the categorisation of cognitive-linguistic abilities exhibited by the students throughout the implementation of the designed unit, as well as analysing the expressions used by them from the communicative perspective; this analysis is born from the upcoming and growing concern of bilingualism implementation in Colombia and Latin America. The methodology used follows an interpretative-qualitative analysis with an inductive analysis approach, analysing the collected information during the didactic implementation in recordings, products developed by students and class diaries from a sample of 25 and 19 students belonging to two private secondary schools located in Cajicá and Bogotá, Colombia. The document presents the reflections arisen from the analysis categories built to assess the collected information: socio-linguistic abilities, communication of ideas in both L1 and L2, the conceptual, social, epistemological and didactic aspects of knowledge.

It was found a close link between the L2 proficiency and the depth of the explanations elaborated by the students, enabling the more competent students in L2 to communicate better using the scientific language and getting to more complex explanations. Moreover, the implementation re-dimensioned the content perspective applied by some teachers when using the CLIL approach, placing bilingualism in the Science classes in a dimension distant from transmitting information, being a medium that fosters communicative and explanatory processes by nurturing different cognitivelinguistic abilities.
\end{abstract}

Keywords: CLIL, bilingualism, construction of explanations, language, science teaching.

1 Este artículo se deriva de la tesis de maestría de los autores, realizada para optar por el título de Magister en Docencia en las Ciencias Naturales, adscrita al departamento de física de la Universidad Pedagógica Nacional.

2 Universidad Pedagógica Nacional, Colombia. mauricio.mancipe452@gmail.com.

3 Universidad Pedagógica Nacional, Colombia. cynthimarce@hotmail.com. 


\section{Resumen}

El presente trabajo aborda la problemática en torno a la construcción de explicaciones de fenómenos naturales a lo largo de la clase de ciencias en contextos bilingües (español (L1)-inglés (L2)), donde se analiza el rol del lenguaje, su significado y su reconfiguración desde dos miradas: la dinámica comunicativa y la dinámica explicativa. Para llevar a cabo dicho análisis, el presente texto se centra en la categorización de las habilidades cognitivo-lingüísticas de los estudiantes durante los distintos momentos de la implementación didáctica, así como en analizar las expresiones usadas por ellos desde lo comunicativo. Este análisis nace de la creciente preocupación frente a la implementación del bilingüismo en Colombia y Latinoamérica. La metodología de trabajo es de tipo cualitativa-interpretativa y utiliza para el análisis instrumentos de recolección de datos como grabaciones de clase y diarios de clase de una muestra de veinticinco y diecinueve estudiantes pertenecientes a dos instituciones privadas de educación básica y media en Cajicá y Bogotá D.C., Colombia. Las siguientes categorías se describen a profundidad: habilidades cognitivo-lingüísticas, comunicación de ideas en la L1 como en la L2, los aspectos conceptuales, sociales, epistemológicos y organizacionales-didácticos del conocimiento.

Ante esto, se encontró una estrecha relación entre la competencia en la segunda lengua (L2) y el nivel de profundidad en las explicaciones construidas, ya que a los estudiantes con mejor dominio del idioma se les facilita usar mejor el lenguaje científico y llegar a construcciones más complejas. Por otra parte, este trabajo redimensionó la perspectiva del contenido implementada por maestros cuando usan el enfoque CLIL (por sus siglas en inglés de Aprendizaje Integrado de Contenido y Lengua), situando al bilingüismo en las clases de ciencias en una dimensión epistemológica y pedagógica diferente a la de adquisición de vocabulario y expresiones en la L2, mostrándose como un medio que favorece los procesos comunicativos y explicativos al potenciar diferentes habilidades.

Palabras clave: bilingüismo, CLIL, construcción de explicaciones, enseñanza de las ciencias, lenguaje.

\section{Introducción}

En medio de las dinámicas globalizadoras del mundo, el lenguaje cobra gran relevancia para comunicarse con otros, además de intensificar procesos económicos y políticos (García, 2009) que han llevado a la educación a expandir las miradas pedagógicas a incluir elementos de índole cultural en sus reflexiones. Resulta relevante analizar las dinámicas que se dan en el proceso explicativo en ciencias considerando su relación con la comunicación de ideas y el diálogo de significados, por ello es necesario tener en cuenta el papel del lenguaje en este proceso basándose en la construcción de explicaciones como eje que articula la propuesta de aula con el proceso de aprendizaje de los estudiantes en un contexto bilingüe.

Del mismo modo, se identifica una preocupación por la enseñanza de las ciencias en el contexto bilingüe relacionada con la comprensión y sentido que se le otorga al bilingüismo en los lineamientos curriculares en Colombia, desde los cuales se evidencia un uso inadecuado del término bilingüismo para orientar procesos de enseñanza (Archila, 2013; Bahamón, 2009; Buck, 2000). Lo anterior se manifiesta en formar al estudiante en la adquisición de una segunda lengua, pero no en permitirle ser competente y capaz de expresar sus ideas en dos lenguas de manera simultánea. Así como la prelación de la adquisición de vocabulario y no la construcción de explicaciones, lo cual desdibuja el objetivo de la enseñanza de otras asignaturas.

Esto exhibe lo que algunos maestros hacen en el aula, diferente a lo que proponen los estándares de educación en Colombia, los cuales propenden por un desarrollo de competencias tales como el uso de la segunda lengua en contexto, en este caso la clase de ciencias naturales. Es decir, lo que está en los estándares no es exactamente lo que se lleva a cabo en el aula, por lo cual este trabajo se centra en proponer estrategias mediante las cuales se logre poner en diálogo lo escrito y lo realizado con los estudiantes. Para esta reflexión, se hace uso de las habilidades cognitivo-lingüísticas como elementos de análisis de la dinámica explicativa en el proceso fenomenológico de construcción de explicaciones en ambientes bilingües.

\section{La educación bilingüe y la enseñanza de las ciencias}

Las preocupaciones identificadas en relación con el uso del concepto bilingüismo en los 
lineamientos curriculares de Colombia se relacionan con las falencias percibidas en la práctica del maestro documentadas por diversos investigadores (Suárez, 2013; Alarcón, 2002; Snow, 1999) que suelen caer en la enseñanza del inglés a través de la clase de ciencias sin considerar la construcción de explicaciones, otorgando un privilegio a la enseñanza de la segunda lengua frente a los otros procesos de enseñanza. En este sentido, se antepone el uso de didácticas propias de la enseñanza de la segunda lengua sin tener en cuenta el conocimiento adquirido en ciencias, por lo cual se desconoce que los objetos de estudio distintos requieren formas de conocer distintas. No es pertinente enseñar ciencias bajo metodologías destinadas al aprendizaje de una segunda lengua, ya que la enseñanza bilingüe implica la formación de individuos bilingües (De Mejía, 1998).

Por ello, el bilingüismo en la enseñanza de las ciencias debe reconocer el adecuado análisis del contexto del sujeto con base en tres relaciones fundamentales: el uso lingüístico, la relación entre lenguaje y conocimiento, y los aspectos culturales. Las relaciones entre el lenguaje y la ciencia son fundamentales para entender la postura en la enseñanza de las ciencias en segunda lengua, así "la ciencia se reconoce como una disciplina altamente comunicativa, donde el lenguaje es central en la naturaleza colaborativa del discurso científico" (Jarrett, 1999, p.7), lo anterior considerando que la ciencia tiene lo que se ha denominado como "su propio lenguaje" dada la complejidad de símbolos, íconos, expresiones y formas de hablar propias. Infortunadamente, estas características propias del lenguaje de la ciencia pueden verse impedidas cuando los estudiantes no logran comunicar sus ideas y darles significado, lo cual es particularmente notorio cuando se enfrentan a clases de ciencias en las que el proceso de enseñanza se da en una segunda lengua, como el inglés.

Sin embargo, teniendo en cuenta la concepción de aulas bilingües planteada por el Ministerio de Educación Nacional (2005) en las cuales los estudiantes y los maestros usan simultáneamente dos lenguas o una lengua extranjera, en la enseñanza de las ciencias por ejemplo, se suelen defender estos procesos en los cuales los estudiantes se enfrentan tanto a un cuerpo teórico como a una segunda lengua. Diversas investigaciones (De Mejía, Ordóñez y Fonseca, 2006; Jarrett, 1999; Choi y Kuipers, 2003) presentan la forma en la cual los estudiantes van y vienen entre la lengua extranjera y la nativa para dar explicaciones y comunicar sus ideas, a esto se le conoce como code-switching (alternancia de código, en español), que puede ser definido como "el uso de más de un código o lengua en el curso de un evento discursivo" (Gumperz, 1982, citado por Choi y Kuipers, 2003, p.2). El code-switching se considera relevante ya que el abordaje de las ciencias naturales en ambientes bilingües ha mostrado falencias en el contexto colombiano (De Mejía, 1998), por lo que se pasa más bien a ser monolingües en una lengua extranjera que no todos los estudiantes manejan con alta competencia, además se desconocen los procesos necesarios cuando se construyen explicaciones en ciencias tales como la discusión, los acuerdos y, en general, la interacción necesaria para desarrollar habilidades científicas fundadas en el lenguaje.

De tal manera, para esta investigación es importante tener en cuenta el tipo de bilingüismo predominante en las instituciones en las cuales se llevó a cabo la investigación. A continuación, se presenta dicha caracterización (Tabla 1), de las que se retoman las definiciones de bilingüismo recopiladas por Suárez (2013), citando a Alarcón (2002) y a Snow (1999).

Se evidencia que las relaciones entre el lenguaje y la ciencia son fundamentales para el entendimiento de la postura en enseñanza de las ciencias bilingüe de la cual se parte, pues "la ciencia se reconoce como una disciplina altamente comunicativa, donde el lenguaje es central en la naturaleza colaborativa del discurso científico" (Jarrett, 1999, p.8), considerando que la ciencia tiene lo que se ha denominado "su propio lenguaje" como ya se ha mencionado. Asimismo, se muestra que la caracterización de los tipos de bilingüismo se centra en dos aspectos: los psicológicos y los sociolingüísticos, relacionados con la competencia comunicativa y la edad de los individuos, pero también con los aspectos sociales que influyen en el aprendizaje o adquisición de una segunda lengua (Alarcón, 2002). 
En este orden de ideas, resulta relevante la caracterización previa, especialmente por las condiciones contextuales en las cuales se desarrolla este trabajo. Así, aunque en los dos colegios en los cuales se desarrolla la investigación el estado del proyecto de bilingüismo es diferente, algo en común es que la población de estudiantes se encuentra inmersa en una cultura predominantemente monolingüe en la cual la lengua que domina es la materna, en este caso el español, razón por la cual no se espera formar individuos con un bilingüismo balanceado sino individuos bilingües dominantes, manifestando competencia comunicativa en dos o más lenguas pero con predominancia en una, en este contexto particular la lengua dominante es el español.

Otros dos elementos en los tipos de bilingüismo citados en la tabla que se observan en este trabajo son el de la edad en la cual se da el proceso. En el contexto del Colegio Emmanuel d'Alzon (G1) un porcentaje considerable de la población suele presentar exposición al inglés a edad temprana, pero también se encuentra una exposición tardía en algunos estudiantes; por su parte, en el Colegio San José de Cajicá (G2) predomina el bilingüismo tardío.

Tabla 1. Definición sobre tipos de bilingüismo.

\begin{tabular}{|c|c|}
\hline \multicolumn{2}{|r|}{ DE ACUERDO CON LA EDAD DE EXPOSICIÓN A LA SEGUNDA LENGUA } \\
\hline Bilingüismo Temprano & $\begin{array}{l}\text { Cuando el contacto con la segunda lengua se da de forma simultánea antes de los tres años de } \\
\text { edad. }\end{array}$ \\
\hline Bilingüismo Tardío & El aprendizaje del segundo sistema lingüístico se da posterior al desarrollo de la lengua materna. \\
\hline \multicolumn{2}{|r|}{ DE ACUERDO CON LA ORGANIZACIÓN COGNITIVA } \\
\hline Bilingüe Compuesto & $\begin{array}{l}\text { - El que tiene una sola representación cognitiva para dos expresiones equivalentes. } \\
\text { - No detecta diferencias conceptuales marcadas en las dos lenguas, sino que necesita de las dos } \\
\text { lenguas para pensar y comunicarse. }\end{array}$ \\
\hline Bilingüe Coordinado & $\begin{array}{l}\text { - Aquel con una representación separada para dos expresiones equivalentes. } \\
\text { - Sin interferencia o mezcla. Dos sistemas lingüísticos paralelos. } \\
\text { - Dos significantes, dos significados. }\end{array}$ \\
\hline \multicolumn{2}{|r|}{ DE ACUERDO CON LA COMPETENCIA COMUNICATIVA } \\
\hline Bilingüe balanceado & Si el individuo posee una "competencia equivalente" en ambas lenguas. \\
\hline Bilingüe dominante & $\begin{array}{l}\text { Si el individuo tiene una competencia superior en una de las dos lenguas generalmente en su } \\
\text { lengua materna. }\end{array}$ \\
\hline \multicolumn{2}{|c|}{ DE ACUERDO CON NIVELES DE PROFICIENCIA EN CONTEXTOS ESPECÍFICOS } \\
\hline $\begin{array}{l}\text { Bilingüe con Habilidades } \\
\text { Comunicativas Interpersonales } \\
\text { Básicas }\end{array}$ & $\begin{array}{l}\text { Quienes logran una fluidez en su L2 pero no pueden manejarla en una situación académica que } \\
\text { implique una abstracción. }\end{array}$ \\
\hline $\begin{array}{l}\text { Bilingüe con Proficiencia } \\
\text { Lingüística Cognitivo Académica }\end{array}$ & $\begin{array}{l}\text { Quienes además de una fluidez comunicativa pueden manipular su L2 en situaciones académicas } \\
\text { descontextualizadas. }\end{array}$ \\
\hline \multicolumn{2}{|r|}{ DE ACUERDO CON EL AMBIENTE SOCIAL } \\
\hline Inmersión lingüística & $\begin{array}{l}\text { Bilingüismo simultáneo en ambiente natural, que consiste en el aprendizaje de la segunda } \\
\text { lengua al mismo tiempo que la primera o con algún retraso, pero desde la primera infancia y al } \\
\text { interior de su grupo familiar. }\end{array}$ \\
\hline \multicolumn{2}{|c|}{$\begin{array}{l}\text { Bilingüismo desde el aprendizaje consecutivo familia escuela, en la que el niño, aunque ha crecido en una familia monolingüe, } \\
\text { ingresa en una escuela que está enfocada en la enseñanza de L2. }\end{array}$} \\
\hline Sumersión & $\begin{array}{l}\text { Bilingüismo a través del contacto directo y sostenido con una sociedad en la que se utiliza esa } \\
\text { lengua. }\end{array}$ \\
\hline $\begin{array}{l}\text { Aprendizaje de L2 en ambientes } \\
\text { educativos }\end{array}$ & emas de educación bilingüe. \\
\hline
\end{tabular}

Fuente: Suárez, J. (2013). 
Lo anterior es de alta importancia ya que como se expondrá posteriormente el trabajo muestra cómo ciertas habilidades son desarrolladas mejor en L1 (español) o L2 (inglés) dependiendo del proceso cognitivo que se lleva a cabo.

Existen distintos enfoques desde los cuales se han buscado establecer relaciones entre el lenguaje y el aprendizaje, por lo cual se adopta para la presente investigación el enfoque de la enseñanza de contenidos disciplinares en segunda lengua denominado CLIL (por sus siglas en inglés de Aprendizaje Integrado de Contenido y Lengua), teniendo en cuenta que la enseñanza se lleva con estudiantes cuya lengua materna no es el inglés. Sin embargo, la mirada de CLIL en relación con el aprendizaje de las ciencias es limitada por parte de algunos maestros a la transmisión de los productos de la ciencia, tales como leyes, teorías, algoritmos, procedimientos, etc., mirada que es reforzada por la mera adquisición de vocabulario y expresiones que si bien fortalecen la adquisición de L2, limitan el proceso de construcción de explicaciones en ciencias distanciándose del fin de CLIL: desarrollar habilidades cognitivas de pensamiento desde el aprendizaje del contenido y la adquisición de una segunda lengua (Meyer, 2010). Lo anterior ha sido observado por parte de los maestros investigadores en su experiencia como docentes bilingües de ciencias naturales. Construir dichas explicaciones implica una reevaluación constante de las ideas individuales fundadas en la socialización y reestructuración de conceptos -entendidos éstos como formas sociales de organización del discurso en el aula - por lo que no es posible dicha reestructuración cuando se enseña una ciencia acabada (que no evoluciona) que nada más debe adquirirse.

Es así como se retoman algunos postulados del enfoque CLIL en el marco del lineamiento de las $4 \mathrm{C}$, especialmente los referentes a los procesos comunicativos, pero distanciándose de la mirada de contenido adoptada por algunos maestros, y reafirmando lo que el enfoque plantea en torno al contenido. Este modelo de las $4 \mathrm{C}$ integra los cuatro componentes principales de CLIL: comunicación, contenido, cultura y cognición. Se presenta como un modelo no jerárquico en el cual hay una interdependencia entre todas las áreas, pero la comunicación es la que permite el diálogo entre cada dimensión del modelo (Zydatib, 2007). Coyle, Hood y Marsh (2010) reafirman que el lineamiento del modelo es "construido sobre la premisa que la cualidad de CLIL es dependiente en el entendimiento y enfoques de operacionalización, los cuales no se encuentran únicamente en los repertorios tradicionales de enseñanza de contenido o de lenguaje" (p.549). Los componentes del modelo se muestran a continuación:

- Contenido: hace referencia al aprendizaje de las temáticas. Es muy conveniente que los profesores vean el contenido en términos de conocimiento, habilidades y entendimiento, ya que esta idea garantizará un proceso de aprendizaje más profundo; por lo tanto, la visión que se propone es de la construcción de explicaciones, y se relaciona con el planteamiento original de CLIL, donde "el contenido no aborda solamente la adquisición de conocimiento y habilidades, se trata de que el estudiante cree su propio conocimiento y desarrolle sus habilidades" (Coyle et al., 2010, p. 16), así, se parte de la postura desde la cual los estudiantes, en un evento o situación que se les presenta o que desean abordar, hacen una descripción detallada, construyen el fenómeno y producen explicaciones que les permitan comprender lo observado. Cabe resaltar que este proceso es tanto individual como colectivo y se lleva a cabo a partir del lenguaje.

- Comunicación: los estudiantes usan la segunda lengua en vez de aprenderla únicamente desde la gramática, y sirve como vehículo para el aprendizaje. CLIL integra contenido $\mathrm{y}$ aprendizaje haciendo estos dos aspectos complementarios, ya que los estudiantes están usualmente involucrados en situaciones que les permiten interactuar de forma colectiva, construir conocimiento y desarrollar sus competencias comunicativas en segunda lengua.

- Cognición: los estudiantes, al estar involucrados en actividades que les permiten construir nuevas comprensiones, usan habilidades 
de pensamiento complejo desarrollando continuamente sus procesos cognitivos y adquiriendo conocimiento al mismo tiempo (integración de contenido y lenguaje). Siempre están enfrentados a situaciones para desarrollar nuevas habilidades como la descripción, explicación, capacidad de relacionar variables, entre otras, que pueden emplear en muchas ocasiones de su vida cotidiana.

- Cultura: se espera que los estudiantes desarrollen un profundo entendimiento del otro y de sí mismos por medio de actividades que les ayudan a comprender las similitudes y las diferencias entre las culturas, a través del uso de materiales auténticos, leyendo sobre otros países y mediante distintos currículos conectados entre sí. Los profesores pueden extender el contenido a diferentes contextos y culturas, guiando a los estudiantes hacia un conocimiento más pluricultural, un sistema de relaciones entre elementos tales como la ciencia, el arte, entre otros, que interactúan entre sí, asumiendo que "el hombre es un animal suspendido en entramados de significación que él mismo ha tejido" (Max Weber, citado por Elkana, 1983, p.279). A esto se le llama cultura.

Uno de los elementos más valiosos del enfoque CLIL es la posibilidad que abre al considerar el contexto particular del estudiante, de reconocerlo dentro de una pluriculturalidad enfocada a la construcción de explicaciones de forma individual y colectiva; esto resulta de gran relevancia para la investigación, pues el enfoque de enseñanzaaprendizaje característico que se asume es la construcción de explicaciones.

\section{El lenguaje}

El lenguaje es parte esencial para la comunicación entre los seres humanos, y en este sentido el dominio de los elementos esenciales del habla es indispensable y de gran significación en el desarrollo individual y colectivo. Desde el punto de vista de la sociolingüística y de las teorías de la comunicación, se ubica el lenguaje en un contexto social y se establecen correlaciones entre el comportamiento lingüístico y el contexto sociosituacional. Los procesos de construcción de explicaciones están directamente sujetos al contexto de los estudiantes, pues incide en las formas de organizar la experiencia en sus esquemas semánticos y cómo logran expresarlas, ya sea de manera verbal o no verbal (escrita, quinésica, paralingüística, proxémica o cronémica). Estas formas o modos de comunicación de los estudiantes permiten el intercambio, la producción y la reconstrucción de significados, los cuales se configuran en una semiósfera o universo de significados (Lotman, 1996).

Desde las posturas de Guidoni (1990) y Lemke (1997), se asume que el lenguaje es un modo de comunicar, construir y plantear ideas, cuestionamientos y significados que conjuga tanto el conocimiento obtenido por el sujeto sobre su realidad como la experiencia y las vivencias en los procesos de aprendizaje. Por tanto, los campos mencionados permiten orientar y dar cuenta de las situaciones de contacto lingüístico y cultural en el contexto de la educación en ciencias en la segunda lengua, pues estas pueden determinar no solo el código o los símbolos sino también los usos del lenguaje y las pautas lingüísticas, comunicativas y cognitivas vigentes en una comunidad determinada, las situaciones comunicativas y los eventos de habla que se ejecutan en ellas, las prácticas discursivas y los modos de hablar, así como el reconocimiento y evaluación de tales prácticas.

En el aprendizaje de las ciencias, un aspecto importante es la apropiación de su lenguaje, proceso que está asociado a nuevas formas de ver, pensar y hablar sobre los hechos, sobre las distintas formas cotidianas de observar, pues a través del lenguaje los estudiantes acceden a una cultura diferente: la cultura científica. El aprendizaje de la ciencia implica aprender a hablar en el código propio de ésta, utilizarlo al leer y escribir, al razonar, al resolver problemas durante las actividades realizadas en la clase y en la vida cotidiana. En este sentido, comunicarse en ciencias implica observar, describir, comparar, clasificar, analizar, discutir, hipotetizar, teorizar, cuestionar, decidir, concluir y enseñar por medio de un idioma único, complejo y abstracto. 
A lo largo de la evolución del análisis discursivo se han implementado diferentes tipos de elementos para estudiar y analizar lo que los estudiantes y los profesores dicen durante una clase, estos permiten categorizar aquellos aspectos que se ponen en juego en la interacción dentro del aula de clases, así, para llevar a cabo dicho análisis, se retoma lo propuesto por la Secretaria de Educación Distrital de Bogotá (2007) en el documento "Colegios Públicos de excelencia para Bogotá. Orientaciones curriculares para el campo de Ciencia y Tecnología", relacionando los elementos propuestos para el proceso de comunicación basados en el lenguaje y el pensamiento, además de los procesos de modelización requeridos en el aprendizaje de las ciencias. Dicho documento plantea que el proceso implica un desarrollo mental complejo y necesita las relaciones del lenguaje y la comunicación de ideas que se dan en los estudiantes.

Los estudiantes explican los fenómenos naturales de su entorno, haciendo uso de unos conocimientos previos y de otros nuevos aprendidos en la escuela, de tal manera que puedan construir nuevas relaciones entre estos conocimientos y llegar a aplicarlos en otros contextos. (SED, 2007, p. 54).

Las habilidades cognitivo-lingüísticas permiten establecer y confirmar la complementariedad entre los procesos comunicativos, de construcción de explicaciones y de desarrollo del pensamiento, ya que como lo plantea Vigotsky "el pensamiento requiere del lenguaje y el lenguaje del pensamiento" (Álvarez, 2010, p.23). A medida que el sujeto desarrolla habilidades del pensamiento más complejas, su proceso comunicativo se complejiza y la construcción de explicaciones se puede llevar a cabo desde su experiencia diaria (conocimiento cotidiano). Se ha establecido que este proceso está directamente relacionado con la apropiación de un discurso que signifique la experiencia del sujeto, el cuál puede verse reflejado en algunos aspectos como: qué se dice, en qué contextos se dice, los diagramas que utilizan los maestros y los estudiantes, qué ecuaciones se expresan y se representan, qué gráficos se interpretan, qué demostraciones se realizan (Figura 1).

Lo anterior se conocen como formas multimodales de expresión, aquellas modalidades semióticasmúltiplesquecaracterizanla comunicación y pueden visualizarse desde el lenguaje oral, escrito, las imágenes y las acciones. La representación que viene dada en el discurso del sujeto se manifiesta como un proceso por el cual el evento, la idea o el concepto es capturado y organizado en alguna forma por el significado, no puede ser percibido como un objeto real en tanto es una entidad abstracta establecida en el colectivo basado en un mismo sistema de reglas o códigos (Lemke, 1997; Candela, 1999). Sin embargo, la interpretación de un significado puede ser otro significado en otro

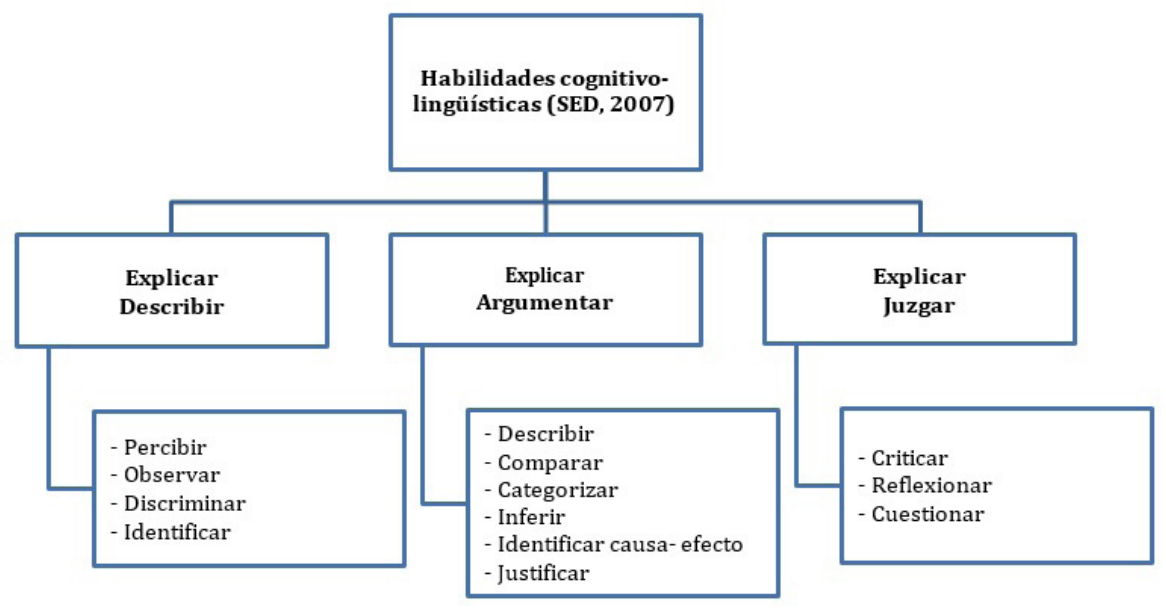

Figura 1. Habilidades cognitivo-lingüísticas del proceso explicativo (en SED, 2007). 
sistema semiótico (contexto diferente), por lo cual se establecen dos tipos de significados: los significados denotativos que señalan o identifican características de un evento y los significados connotativos que incluyen sensaciones, emociones o sentimientos que son provocados por el evento (Jaipal, 2011; Lemke, 1997).

Jaipal (2011) parte de la semiótica social de Lemke (1997) y propone cuatro niveles del análisis del discurso en ciencias, en los cuales se elaboran y se reconfiguran los significados desde los aspectos conceptuales, sociales, pedagógicos y epistemológicos. Estos niveles son:

- Aspectos conceptuales: indican los significados denotativos expresados por medio de formas de expresarse sobre características del concepto.

- Aspectos sociales: se enfocan enla interpretación de los significados desde la postura del sujeto según los roles de los participantes, se puede llegar a negociar el significado en el cual la validación se da en una triada entre el sujeto, el evento y la interpretación en sí.

- Aspectos organizacionales-didácticos del significado: se refiere a los significados comunicados por medio de las modalidades semióticas (formas de expresar) empleadas por los maestros en el desarrollo de las actividades, por ejemplo, las actividades dirigidas como preguntas, o cuando emplean recursos audiovisuales para presentar el evento.

- Aspectos epistemológicos: comprenden la interpretación del significado para dar cuenta de la naturaleza del conocimiento científico que siempre está en continua reestructuración, conforme a los diversos procesos de razonamiento que se estén llevando a cabo (deductivos, inductivos y abductivos).

\section{Metodología}

La investigación se realiza en dos colegios de educación básica y media: el primero (G1), ubicado en un barrio de clase media de la localidad de Suba en el suroccidente de Bogotá D.C.; el segundo (G2), ubicado en un municipio cercano a Bogotá D.C. La investigación se desarrolla en los grados sexto y séptimo, con una población de veinticinco estudiantes en el primer colegio y un programa de Aprendizaje de L2 en ambientes educativos (Tabla 1), pero sin responder a un currículo bilingüe o internacional, mientras que se trabaja con diecinueve estudiantes en el segundo colegio asociado al programa bilingüe internacional de Cambridge.

Para abordar el problema planteado se elige un enfoque de investigación interpretativo, con una perspectiva cualitativa, empleando el estudio de un caso múltiple con dos unidades de análisis. El objetivo principal es comprender las dinámicas presentes en los procesos que intervienen en el aprendizaje en ciencias y luego caracterizarlas para analizar la construcción de explicaciones del estudiante en la clase de ciencias bilingüe específicamente acerca de la temática ecosistema.

Este tipo de metodología adopta un modelo de análisis inductivo para desarrollar categorías de análisis conceptuales que ilustran, ratifican y describen los presupuestos teóricos acerca de la educación bilingüe en ciencias (Barrio et al., 2012). La información recolectada corresponde en su mayoría a grabaciones de cada sesión de clase, productos elaborados por los estudiantes y diarios de clase de los docentes bajo un ambiente de aprendizaje lo más natural posible, con el fin de dar cuenta de las dinámicas reales que son objeto de estudio en este trabajo. Dicha información se examina y clasifica bajo unas categorías de análisis que surgieron a partir de un ejercicio de sistematización realizado por parte de los investigadores, estas son: dinámicas comunicativas, dadas desde las modalidades enunciativas y semióticas usadas en el aula de clase; dinámicas explicativas, aquellas que interfieren y jerarquizan los procesos de producción de explicaciones, como la intencionalidad de las preguntas; finalmente, el lenguaje-significado y su reconfiguración, que giran en torno al lenguaje como significado, caracterizándose desde su origen, su modo de expresión o recursos semióticos y el proceso que se da en el sujeto. Para tal fin, se llevan a cabo los siguientes procesos: 
- Se emplea la técnica de análisis de secuencias narrativas y argumentativas que describen lo que los estudiantes manifiestan de forma oral y escrita en inglés sobre el fenómeno de estudio, ecosistemas, buscando presentar de forma fidedigna incluso los posibles errores conceptuales o gramaticales.

- Se analizan las estrategias de explicación a las que acuden los estudiantes empleando diferentes recursos semióticos y formas de expresar, lo cual se analiza desde la dinámica explicativa.

- A través de las secuencias narrativas y argumentativas, se describen las interacciones en un ámbito comunicativo exhibidas por los estudiantes, insumos que son la base de los hallazgos presentados en este trabajo.
La intervención didáctica -amazing interactions within ecosystems- se ha estructurado en cuatro momentos (Figura 2) que representan las etapas del proceder fenomenológico asociadas a unas habilidades cognitivas y cognitivolingüísticas desde el enfoque CLIL, las cuales permiten analizar la progresión de la construcción de explicaciones sobre el objeto de estudio. De igual manera, se demarca el análisis de las vivencias, la interpretación de la realidad y la construcción de explicaciones que lleva a cabo el sujeto con respecto al fenómeno. Se buscó que los estudiantes tuviesen una participación constante con el fin de evaluar la dinámica comunicativa desde diferentes elementos y su relación con la construcción de explicaciones desde el diálogo de significados.

La metodología descrita se ha seleccionado en tanto responde a los intereses de los investigadores,

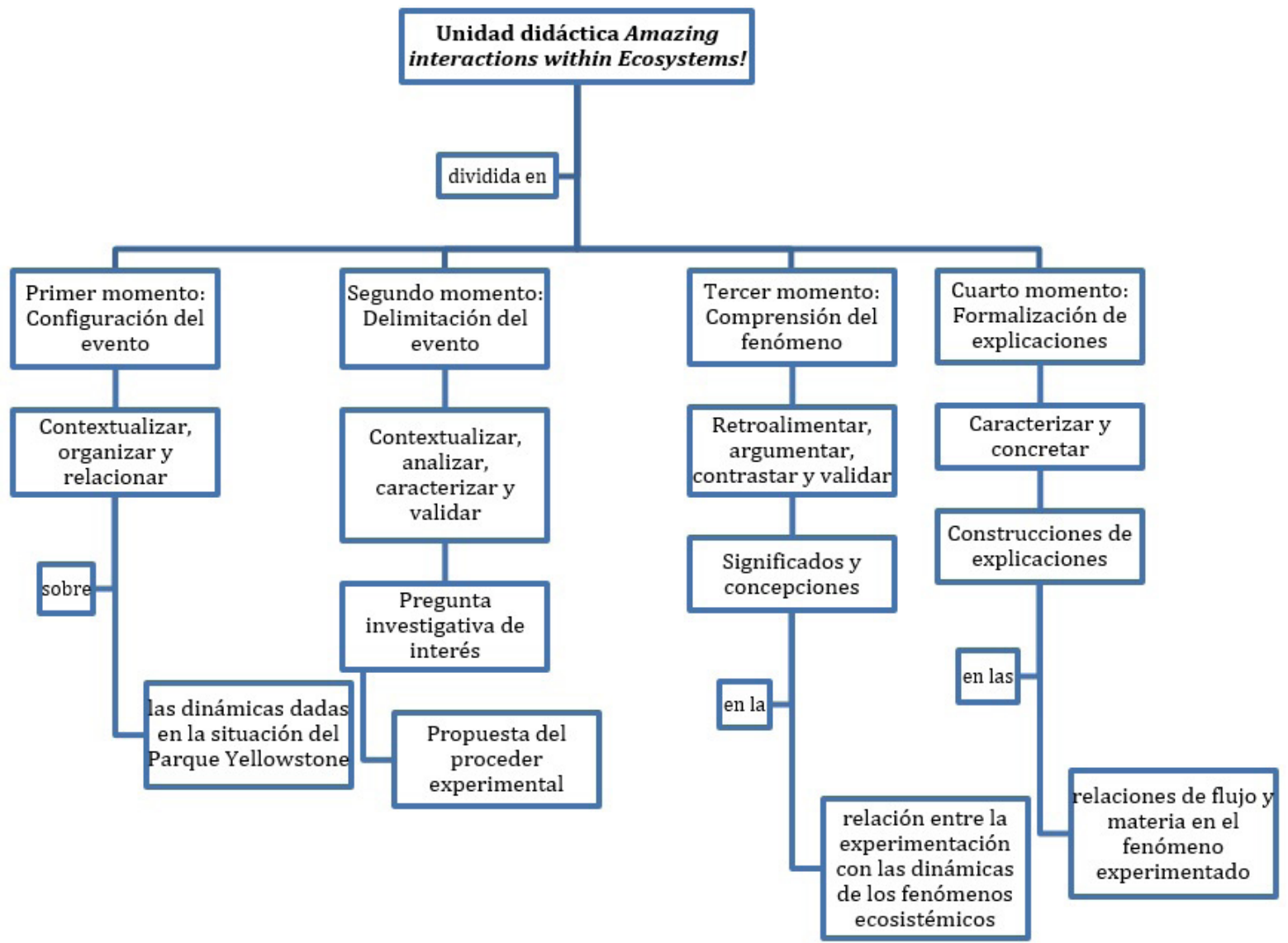

Figura 2. Situaciones de la implementación fenomenológica (elaboración propia). 
nace de la labor de ambos docentes en el campo de la enseñanza bilingüe y permite reconocer dinámicas con estudiantes reales y contextos locales. Cabe señalar que, desde la observación en el aula, la recolección de información in situ y la sistematización, permitieron lograr el objetivo del presente trabajo. Esta metodología, además, aporta al enfoque de construcción de explicaciones en ciencias desde el proceder fenomenológico en el cual se sitúa la investigación, haciendo hincapié en las categorías de análisis construidas y enfatizando en las habilidades cognitivo-lingüísticas como se verá en el siguiente apartado.

\section{Discusión de los resultados}

Esta investigación analiza y caracteriza el papel del lenguaje en las dinámicas de la clase de ciencias desde lo comunicativo, lo explicativo y el lenguaje como significado. El análisis está estructurado en unos momentos o fases a los cuales se asocian habilidades cognitivas y cognitivo-lingüísticas, en los que se enmarca el estudio de las vivencias, la interpretación de la realidad y la progresión de la construcción de explicaciones que lleve a cabo el sujeto en dos miradas de los ecosistemas: las relaciones a nivel poblacional y los efectos de las interrelaciones entre los factores en los ecosistemas.

\section{Primer momento: configuración del evento}

Este primer momento analiza el uso de la lengua materna (L1) y la segunda lengua (L2) por parte de los estudiantes, además de caracterizar la incidencia de las relaciones lenguaje y conocimiento científico escolar, se aclara que en las instituciones independientemente de su programa en lengua extranjera es permitido hacer code-switching entre L1 y L2 en las clases de contenido. Las primeras modalidades enunciativas que los estudiantes expresaron en su trabajo grupal (tres niños por grupo) fueron del tipo interrogativo parcial, relacionadas con la preocupación de escribir correctamente lo que opinan en inglés (L2), estas dudas surgieron de la presentación y contextualización del evento a estudiar: el efecto de las variaciones poblacionales en un ecosistema -Yellowstone Park and the reintroduction of the wolves-. A pesar del nivel de competencia oral en la segunda lengua, las actividades resultan favorables ya que los estudiantes adquieren vocabulario específico y lo van usando en sus intervenciones, por lo que "aprenden lengua mientras se les enseña contenido" (Mariño, 2014), asimismo las actividades se convierten en una posibilidad más que en un impedimento porque promueven el interés en los estudiantes (engagement) y los invita a participar activamente.

La intencionalidad comunicativa en este caso es identificar el evento presentado, se basa en la necesidad de llegar a acuerdos entre los estudiantes, busca validar la comprensión de la situación abordada y que las ideas desde las cuales se va a partir para trabajar el resto de las actividades sean comunes en el grupo. Así, este proceso comunicativo donde el profesor pregunta, los estudiantes preguntan y surgen respuestas y discusiones, permite aclarar posibles elementos que enriquecen la construcción del fenómeno, por lo cual el individuo plantea sus ideas, se cuestiona y re-configura sus posiciones a través de la colectividad, como es el caso de hacer un cuestionamiento a modo de hipótesis del evento, reflejando otro nivel de organización del fenómeno.

Los estudiantes con mayor competencia en L2 participan de forma frecuente y con ideas más elaboradas. Se evidencia en ambos grupos una mejoría en las habilidades de nivel abstracto (Tabla 2) donde es necesario seleccionar con precisión la información, ordenarla mediante relaciones que ellos consideran lógicas y, por lo tanto, comunicar ideas particulares. Puede considerarse que el lenguaje toma un papel que va más allá de la mera comunicación de ideas y se constituye en un proceso de construcción basado en la organización de la información, la jerarquización y las interrelaciones entre diferentes sucesos del proceso de construcción de explicaciones (Lemke, 1997). También hay una marcada diferencia en el ámbito comunicativo que les permite a los estudiantes del G2 avanzar más rápido y cuestionarse más, mientras que la dinámica explicativa del G1 es pausada, detallada y se centra en asegurar la comprensión de todos los estudiantes antes de avanzar. 
Tabla 2. Habilidades cognitivo-lingüísticas del proceso explicativo.

\begin{tabular}{|c|c|c|c|}
\hline Grupo & Expresiones y preguntas & $\begin{array}{l}\text { Habilidad } \\
\text { cognitiva }\end{array}$ & $\begin{array}{l}\text { Habilidad cognitivo- } \\
\text { lingüística }\end{array}$ \\
\hline G1 & $\begin{array}{l}\text { "Miss, how you say in English "los científicos observan?". } \\
\text { "Only one species could do that? How it did?". } \\
\text { "There were overpopulation of animals". } \\
\text { "Several bears left the park because they had no food". } \\
\text { "Because the wolves not eat the deer and elk. The plants were not } \\
\text { fertilization and not grow up". }\end{array}$ & $\begin{array}{l}\begin{array}{l}\text { Observar } \\
\text { Discriminar }\end{array} \\
\text { Identificar detalles } \\
\text { Reconocer } \\
\text { Recordar } \\
\text { Identificar causa- } \\
\text { efecto } \\
\text { Predecir }\end{array}$ & G1: Explicar- describir. \\
\hline G2 & $\begin{array}{l}\text { "They almost got extincted". } \\
\text { "Because of humans, they hunted them". } \\
\text { "They said it was an endangered species." } \\
\text { "Because they were a main predator, and controlled animals that } \\
\text { eat plants". } \\
\text { "Less soil for the rivers, because there weren't trees around so the } \\
\text { roots wouldn't stop the soil moving though the river, there would be } \\
\text { erosion". } \\
\text { "Why the wolves affect the control of populations of big trees?". } \\
\text { "How do wolves affect the rivers?". } \\
\text { "As the population of elks increases too much, when they drink } \\
\text { the water of the river, cause erosion, when they eat the plants they } \\
\text { don't leave them grow and this affects the river, because there are no } \\
\text { plants for beavers to build their dams, that prevent erosion". }\end{array}$ & $\begin{array}{l}\text { Observar } \\
\text { Discriminar } \\
\text { Identificar detalles } \\
\text { Reconocer } \\
\text { Recordar } \\
\text { Identificar causa- } \\
\text { efecto } \\
\text { Predecir } \\
\text { Inferir }\end{array}$ & $\begin{array}{l}\text { G2: Explicar- describir y } \\
\text { argumentar }\end{array}$ \\
\hline
\end{tabular}

Fuente: elaboración propia.

Un mayor desarrollo de la competencia oral en L2 permite una relación de liderazgo, los estudiantes se constituyen como fuentes de validación del conocimiento en tanto que sus compañeros no acuden solamente a los maestros o al diccionario, sino también a ellos, lo cual es un proceso fundamental en la construcción de conocimiento: la interacción entre pares.

\section{Segundo momento: delimitación del evento}

La finalidad es promover en los estudiantes interés y motivación, aterrizando la idea de interacción desde su contexto local, para abordar los ecosistemas y los flujos de materia y energía. De igual manera, se quiere analizar lo que preguntan los estudiantes, en especial los recursos semióticos que emplean teniendo en cuenta los efectos de usar L1 y L2 para presentar sus ideas. También, se busca fomentar el trabajo colectivo en los estudiantes desde la discusión al interior de los grupos como paso fundamental en sus procesos, asumiendo fuertemente la idea de que la comunicación es el eje fundamental de la construcción de significados, por lo que se favorece de manera más personal sin ignorar la dinámica de ir y venir entre lengua materna y segunda lengua. Adicionalmente, esta actividad de socialización del evento busca darles un input a los estudiantes y una participación activa y crítica de su realidad. En el marco del enfoque CLIL, el rich input es clave y se justifica desde el 
abordaje de las situaciones en un contexto próximo y local de los estudiantes.

Ante esto, se logra reflejar en los estudiantes una alternancia comunicativa entre afirmar o tratar de argumentar acerca de las posibles razones por las que se dan las relaciones que han venido identificando en los dos eventos (parque Yellowstone y río Bogotá-humedales artificiales) y generar nuevos cuestionamientos que les permitan retroalimentar constantemente dichas afirmaciones. El efecto directo que tiene esta alternancia de enunciados en los estudiantes es que lleguen a cuestionar aún más sus conocimientos de base e ideas previas, incluso que en algunos casos se llegue a comparar lo que han logrado identificar, relacionar y explicar en las sesiones con el tema de ecosistemas visto en años anteriores.

La comunicación de sus ideas en L2 es un proceso sólido, construyen preguntas usando palabras claves en este proceso tales como las $\mathrm{WH}$ questions y los condicionales como "What would happen if", lo cual favorece la comunicación de sus ideas y la socialización para la construcción de significados (Meyer, 2010; Mariño, 2014). No obstante, las explicaciones dadas aún están en un primer nivel, de tipo descriptivo y narrativo, donde hay un diálogo entre su realidad y el proceso de construcción que están llevando a cabo: retroalimentan y reestructuran sus significados al descartar ideas e incluir otras. Estas explicaciones van encaminadas a la comprensión de los procesos o interacciones que han identificado: la relación del hombre con su entorno, los ecosistemas y su influencia sobre factores económicos (los pobladores de dichos ecosistemas intervenidos), y el rol del gobierno sobre el tratamiento de esos ecosistemas.

Los estudiantes delimitan el evento a través de una pregunta problema y un montaje experimental para que puedan hacer un poco más visible su preocupación conceptual sobre las dinámicas ecosistémicas. La progresión en las habilidades cognitivas empleadas van de lo descriptivo a lo inferencial y clasificatorio de las relaciones observadas en la situación de Yellowstone y del río Bogotá como los efectos de la ausencia de un organismo o la contaminación del agua, por lo que empieza a existir una noción de los ecosistemas desde una perspectiva más holística dejando de lado las relaciones básicas de depredación, competencia, cooperación y alimentación para pasar a cuestionarse sobre efectos a nivel macro de los ecosistemas sin dejar de lado las relaciones entre el organismo y el entorno, y la multidireccionalidad de las dinámicas al interior de un sistema dado, tales como: relación entre la turbidez del agua y la posibilidad de la luz para entrar al fondo del recipiente, y por tanto el efecto sobre el crecimiento de las plantas, el rol de un organismo como las lombrices y todo lo que implica en su supervivencia bajo ciertas condiciones, entre otros.

Como lo han manifestado varios autores (Lemke, 1997; Candela, 1999; Arcá, Guidoni y Mazzoli, 1990) los significados sólo existen y son reales para el sujeto desde sus vivencias, es entonces que en cada momento relacionado con los procesos de aprendizaje, los estudiantes siempre ponen ante sí sus experiencias personales (conocimiento cotidiano), las cuales cambian de sentido cada vez que vuelven a enfrentarse a ellas o a una nueva. Para este caso, la situación del río Bogotá y los humedales artificiales permitió que emergiera nuevamente la necesidad en ellos de compartir y socializar dichas experiencias en L1 y L2, como las ocasiones que han percibido el mal olor al pasar por el salto de Tequendama. Estas vivencias, al ser compartidas, entran a formar parte en el proceso de significación, vinculando aspectos valorativos sobre las situaciones presentadas en donde se hacen juicios de valor, dado que lo estudiado les afecta de manera cercana y se promueve el planteamiento de alternativas de explicación de las interacciones que ocurren al interior de un ecosistema.

\section{Tercer momento: comprensión del fenómeno}

Para este momento se busca identificar el tipo de preguntas que los estudiantes se han formulado y las concepciones de las dinámicas propias del fenómeno, como el análisis del diálogo de significados que se establece teniendo en cuenta los procesos de validación, las fuentes de información, las relaciones de poder en el marco de la socialización 
y el uso de L1 y L2 por medio de la actividad del juego de roles. Es importante reconocer que la dinámica de socialización en este ejercicio excede el diálogo sobre las relaciones en el ecosistema y trasciende, incluyendo elementos axiológicos que ocasionan una respuesta en los estudiantes frente a los planteamientos de sus compañeros. La construcción de significados se dialoga y se constituye en una mirada pluridimensional que alimenta la visión de ciencia en ellos, además de permitirles relacionar más allá de lo vivo y lo no vivo a través del acto comunicativo y de los significados existentes en el contexto local. Se da una incesante constitución y legitimación en el discurso de los estudiantes con una mayor competencia, la cual se ve reflejada con los actos perlocutivos ${ }^{4}$.

Dentro de las diferencias comunicativas es importante mencionar que en el G1 y en el G2 se nota la marcada diferencia de la dinámica del aula por el simple hecho de encontrar diferentes niveles de competencia. Mientras que en el G1 los estudiantes escribieron su intervención en la actividad a modo de preparación, la tradujeron y luego se dio el debate; en el G2 los estudiantes solo leyeron la función de su rol en la discusión, pero no se escribió ni se tradujo. Esto es clave, ya que el tiempo de preparación es mayor en un grupo y los procesos deben hacerse con mayor detalle para asegurar la comprensión y comunicación de las ideas de forma asertiva en todos.

En cuanto al montaje experimental que cada grupo ideó, los estudiantes construyen una serie de preguntas que guían su proceder experimental, aunque algunos son más rigurosos en el proceso que otros. Para analizar este aspecto se revisan algunas de las preguntas planteadas por los grupos, estas preguntas son importantes ya que cuestionarse por el "qué" los pone en un plano de causalidad, el cual los puede llevar a analizar la situación sin preguntarse por el "cómo". Las preguntas planteadas desde el "qué pasaría si..." indagan sobre la variación de las partes en el todo y se responden desde las consecuencias de dicha variación, pero no se preguntan sobre el "por qué" esas variaciones afectan el todo. Los estudiantes

4 Acto que modifica lo existente en una persona. que se cuestionan por el "cómo" amplían su mirada y perspectiva explicativa, no se quedan en lo concreto, sino que van un poco más allá, a una mirada más compleja del fenómeno, no se limitan a un tipo de respuesta predeterminado por el "qué" dado que el grupo responde desde sus nociones y le dan dirección a su formalización.

Es por esto que el "cómo" y el "por qué" dan diferentes tipos de formalizaciones que permiten mirar el proceso y la forma como se relacionan los elementos, pero también permiten comenzar a transitar de lo visible a lo construido en tanto requieren inferir o visibilizar aspectos no perceptibles directamente. En este sentido, ya no solo se habla de un objeto o cualidad sino del centro, es decir de las relaciones, la manera cómo se afectan estas relaciones y cómo se afectan los elementos del ecosistema desde la alteración de la relación. Ejemplos de estas relaciones se evidencian en los productos realizados por los estudiantes y presentados en las tablas ya expuestas donde emergen conceptos como adaptación, ciclo, intercambios de materia y energía.

Del mismo modo, se observa un progreso en las habilidades cognitivo-lingüísticas, pues en sus procesos de construcción de explicaciones se está efectuando una transición de lo simple a lo complejo y de lo que han vivido a lo modelado, ya que los fenómenos inmersos en un ecosistema usualmente son aquellos que no son directamente perceptibles para el sujeto que aprende, por ejemplo cuando expresan que saben que el ciclo del agua está presente en el montaje pero aún no lo han podido visualizar. Lo anterior permite que las habilidades cognitivo-lingüísticas de los estudiantes tengan un avance en su desarrollo, evolucionando a un nivel que ya no es meramente descriptivo o narrativo. Los estudiantes detallan los procesos y las interacciones que pueden ocurrir en el fenómeno por medio de sus supuestos, es este el caso de uno de los grupos, el cual manifiesta que los excrementos purifican la tierra al otorgarle nutrientes que pueden ser aprovechados por la lombriz y la planta.

Este proceder experimental permite que ellos descarten o incluyan información para seguir dándole forma a su explicación según las socializaciones que 
se dan al interior de cada grupo de trabajo, por tanto los estudiantes comprenden que las relaciones no solo son importantes para el ecosistema donde están presentes, sino que afectan a otras especies y entornos. Ya hay visos de una noción un poco más holística, no relacionan únicamente lo vivo con lo no vivo sino las dinámicas del ecosistema con actividades económicas y humanas que son parte de la cultura; el ecosistema se discute desde otros puntos de vista que amplían la visión de organización del todo y las partes de un sistema. Para tal fin, ha sido necesario que empleen habilidades más básicas, las cuales progresivamente fueron reestructurándose como la descripción y discriminación de elementos o relaciones, lo cual es la base para que se analice, se infiera y se planteen juicios y valoraciones. Por ejemplo, los estudiantes expresan la necesidad de que los organismos se adapten a las variaciones del medio para poder sobrevivir, pero además, deben competir entre sí con el fin de obtener los recursos necesarios para la vida.

No obstante, coexiste aún la idea del ecosistema como medio, como recurso que suple las necesidades de lo vivo, a pesar del establecimiento $\mathrm{y}$ reconocimiento de la multicausalidad y multidireccionalidad de las relaciones. Adicional a esto, aún no se configura una idea de ecosistema desde la mirada de la complejidad, pero se evidencia un avance en comprender que hay una circulación de materia y energía a partir de los ciclos tróficos del agua y del nitrógeno, mencionados por algunos grupos en sus avances. En otros grupos, la idea de ciclo y de flujo se encuentra de manera implícita cuando reconocen el rol de los descomponedores para la recirculación de nutrientes en el sistema, lo cual indica que los estudiantes manifiestan ideas como "al variar un solo factor, el balance del todo se afecta".

\section{Cuarto momento: formalización de explicaciones}

Al caracterizarse los niveles alcanzados por los estudiantes en su producción discursiva, basándose en la experimentación llevada a cabo, y enmarcada en el flujo de materia y energía en los ecosistemas, se pudo observar que en la socialización final el estudiante muestra deseo por compartir aquello que ha construido acerca de su proceder experimental y de las conclusiones a las que llegó con el fenómeno, por lo tanto las negociaciones o las validaciones que se dan en medio de la dinámica del aula tienen el propósito de que la explicación sea aceptada, retroalimentada y reestructurada (actos perlocutivos), es por eso que por ejemplo, entre ellos no admiten que alguien hable de "cadenas alimenticias" porque consideran que su uso en los ecosistemas es muy reducido y no abarca toda la problemática que han venido discutiendo y construyendo.

En los informes escritos de la actividad experimental, los estudiantes acuden a un nivel predominantemente descriptivo y narrativo del fenómeno, sus informes incluyen muchas observaciones y narraciones del día a día, en algunos no se presentan elucidaciones profundas que permitan ver un nivel mayor de complejidad. Sin embargo, hay grupos que tratan de dar una explicación inicial de lo observado pero se basan particularmente en relaciones de causa y efecto derivadas de lo que pudieron llegar a observar en sus experiencias, aunque logran generar nuevos cuestionamientos que les permiten retroalimentar constantemente dichas afirmaciones.

Por tales razones, en el cuarto momento de formalización de las explicaciones no se ve un avance significativo en el grupo G1, se asume que la dificultad primordial es el no poder comunicar con mayor asertividad sus ideas en L2 de forma escrita. Los estudiantes se esfuerzan mucho al hacer una intervención: en primer lugar la preparan, ya sea escribiendo lo que quieren decir o preguntando a su compañero sobre cómo se pronuncia "relación, adaptación, extinción o afectar"; posterior a ello, hacen uso continuo del diccionario, muchos afirman que se sienten perdidos si no lo traen consigo a las clases de ciencias naturales, sin embargo se les da importancia a las palabras, como las que se mencionaron anteriormente, porque creen que son palabras fundamentales para poder explicar lo que han percibido de las relaciones que se suscitan en los ecosistemas.

Caso contrario a lo que ocurre en el G2, los estudiantes usaron L1 y L2 para dar cuenta de sus 
construcciones en los informes sin mayor dificultad, ya que las construcciones incluyen elementos teóricos que dan cuenta de un nivel explicativo más que descriptivo, en la mayoría de los grupos hay argumentos y una breve discusión de los resultados que llegaron a observar del fenómeno (Tabla 3). Las participaciones de los estudiantes se muestran tal cual ellos las expresaron.

Tabla 3. Formas de representación de estudiantes y maestros.

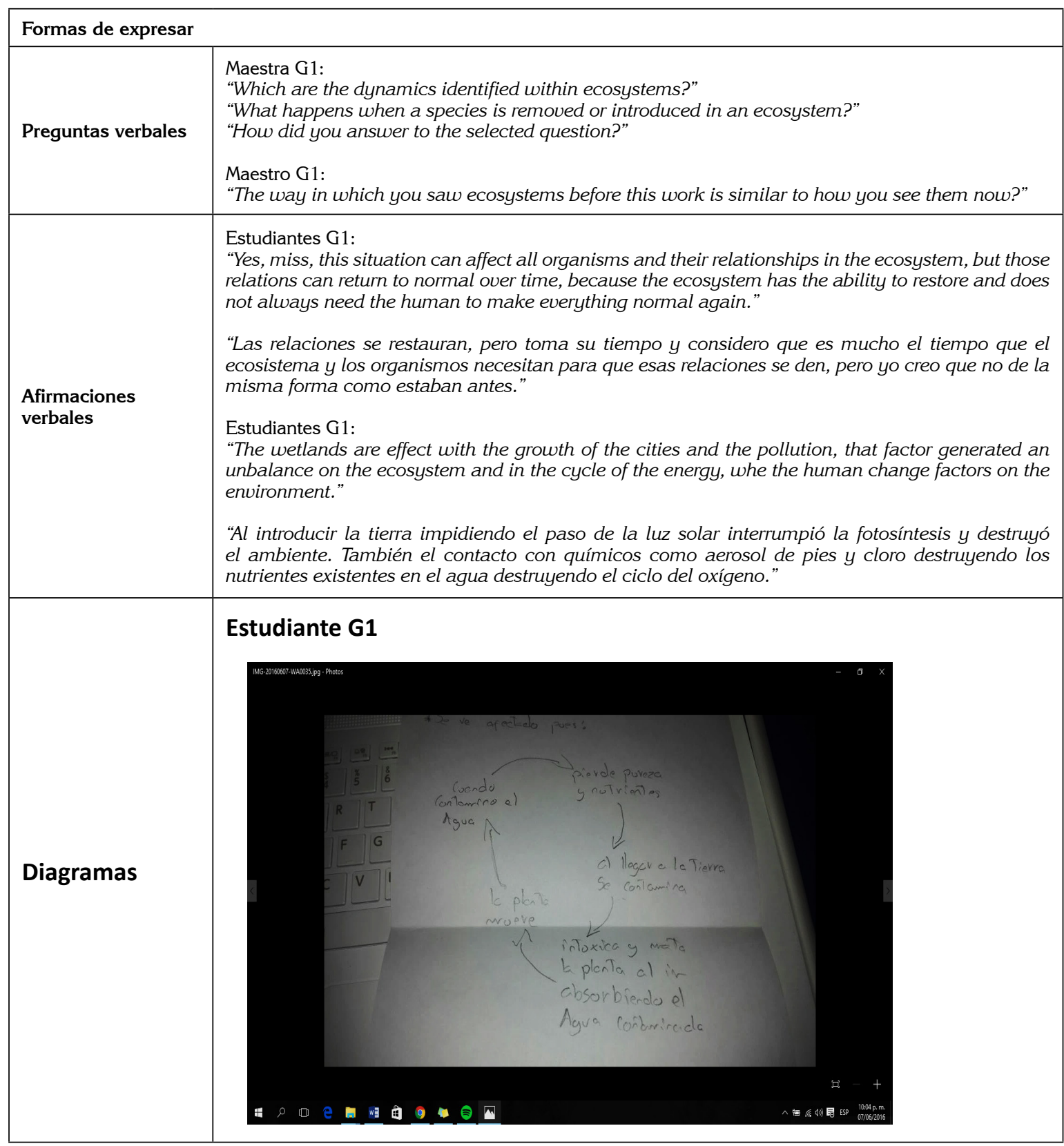

Fuente: elaboración propia. 
En general, la construcción del significado por parte del estudiante se logra en la práctica mediante la socialización del discurso en el colectivo, es por ello que los discursos reflejan los esquemas fundamentales de su realidad, la cual continúa reestructurándose en el acto social y comunicativo. Teniendo en cuenta lo anterior, cada experiencia nueva que se presenta al estudiante permite enriquecer su noción de mundo y su discurso en el aula. Para este momento final de la implementación se encuentran unos significados un poco más elaborados, los cuales enfatizan más en tratar de comprender el fenómeno que se ha venido elaborando acerca de las interrelaciones en los ecosistemas.

Como Candela (1999) enuncia: "El discurso requiere de apropiarse de una forma de construir con palabras el significado de la experiencia" (p.53), así los estudiantes evidencian en su discurso una organización argumentativa de lo que en colectivo han venido construyendo a partir de sus representacionesmentales, debido a quelanaturaleza ya no es completamente estática y ordenada. A pesar de esto, la mayoría no concibe que los elementos constituyentes de los ecosistemas y sus relaciones pueden estar sujetos a cambios en el tiempo, dentro de su noción, parte de los elementos constituyentes de un sistema ecológico son individuos que tienen unas exigencias ambientales y un organismo debe adaptarse a las nuevas variaciones de los factores que en un comienzo puede ser crítico, pero luego logran restaurar sus relaciones. Por otro lado, se evidencian nociones de flujos de materia y energía a partir de ideas como los ciclos de la materia o reutilización de la materia, pero aún con visos de que el medio es un recurso y que está delimitado completamente por lo vivo.

En el proceder de la actividad experimental buscan determinar qué consecuencias puede causar la alteración de uno de los recursos como la luz solar, el agua o el suelo en un organismo específico. Aunque las actividades propuestas en la unidad didáctica aportaron elementos conceptuales $y$ argumentativos en L2, el efecto de su discurso busca la aprobación de los maestros, pues ven en ellos una autoridad de conocimiento sobre la asignatura en tanto que "la autoridad pedagógica se presenta en la relación comunicativa cuando la comunicación de un sujeto (el profesor) sirve de premisa motivacional para las decisiones de otros sujetos (alumnos), los cuales han tenido abierta la posibilidad de negar tal comunicación" (Zamora y Zerón, 2010, p.101).

\section{Consideraciones finales y reflexiones pedagógicas}

Los significados que construyen los estudiantes deben pasar por unos procesos de validación colectiva entre estudiante-estudiante, estudiantemaestro y estudiante-fuentes de información, donde el efecto que más se puede observar en estas dinámicas sociales es la constitución de una autoridad o liderazgo de saberes y de comunicación —en este caso, en L2 entre los sujetos- con el fin de que los estudiantes se apropien de los significados y estos formen parte de la estructura cognitiva del individuo. Lo anterior es importante cuando los estudiantes reflejan los mecanismos mediante los cuales acceden a la información, la incorporan en sus estructuras mentales y eventualmente la aplican para explicar su realidad. Dichos mecanismos hacen referencia al proceso de observación, análisis de la información presentada, construcción del fenómeno y la eventual explicación de los hallazgos de su proceder fenomenológico.

El uso de L1 y L2 cobra otro sentido ya que no es el simple hecho de poseer una ventaja en la competitividad del sujeto al dominar una lengua extranjera, sino que su finalidad va más allá, no se queda solo en la memorización de vocabulario y expresiones en L2 o en la traducción de textos y la replicación de la información presentada en estos, sino que se posiciona en otra dimensión epistemológica ypedagógica, medio que favorece los procesos comunicativos y explicativos al desarrollar diferentes habilidades cognitivo-lingüísticas, tanto en L1 como L2. En un aula bilingüe los estudiantes tienen la capacidad de potenciar dichas habilidades mediante la discusión constante entre pares y con sus maestros, progreso que se ve reflejado incluso en aquellos estudiantes que no son altamente competentes en L2. Este proceso refleja una de las finalidades principales de CLIL, en el cual se lleva a 
cabo un desarrollo de habilidades cognitivas desde el aprendizaje integrado de la construcción del conocimiento en ciencias y lengua.

Expresarse en L2 en las clases de ciencias ya no es obligante en los estudiantes, sino que es complementario a lo que él logre construir en la lengua materna, no obstante este proceso de bilingüismo debe ser continuo en las instituciones educativas y en el aula de clase donde pueda abarcar otros espacios de interés que permitan el desarrollo de un alto nivel de interacción comunicativa, haciendo uso tanto de la lengua nativa como la extranjera, pues el proceso de construcción de explicaciones puede enriquecerse en tanto hayan habilidades particulares de la ciencia que pueden desarrollarse con mayor complejidad en la lengua materna como juzgar, criticar y otras que puedan verse mejor desarrolladas en la segunda lengua tales como describir, inferir y comparar.

Por otro lado, como se mencionó anteriormente, algunos de los docentes y maestros que hacen parte de los proyectos de bilingüismo en instituciones educativas de carácter privado y público del país usualmente son egresados de programas de licenciaturas en inglés o en lenguas extranjeras y son quienes enseñan temáticas en las asignaturas de ciencias, sin embargo uno de los aportes de este trabajo es evaluar la idea que sostiene que los profesores de ciencias naturales no tienen todas la facultades para llevar a cabo estos programas bilingües en cualquier etapa escolar, por lo que permite evidenciar que no solo se vieron potenciadas diferentes habilidades comunicativas y explicativas en los estudiantes sino también en los maestros que diseñaron e implementaron esta propuesta de aula, los cuales a su vez, han tenido toda su formación profesional en el área de las ciencias naturales, invitando a las instituciones educativas que están en dichos procesos y acuden al profesional de la enseñanza del inglés, a capacitar a los maestros de ciencias en el idioma extranjero para que los procesos descritos sean desarrollados de la mejor manera. Con todo lo anterior, se da cuenta de la relación estrecha entre el lenguaje y el explicar en la clase de ciencias, la influencia de la lengua extranjera en la clase y cómo la forma de aproximar a los estudiantes a la ciencia depende en gran parte de la visión del maestro, por lo que el proceder metodológico cobra un papel fundamental.

\section{Referencias}

Alarcón, L. (2002). El fenómeno del bilingüismo y sus implicaciones en el desarrollo cognitivo del individuo. Recuperado de https://www.uv.mx/ cpue/coleccion/n_29/el_fen\%C3\%B3meno_del_ biling\%C3\%BCismo.htm

Álvarez, C. (2010). La relación entre lenguaje y pensamiento de Vigotsky en el desarrollo de la psicolingüística moderna. Revista de Lingüística Teórica y Aplicada, 48(2), 13-32. https://doi. org/10.4067/S0718-48832010000200002

Arcá, M., Guidoni, P. y Mazzoli, P. (1990). El desarrollo del proceso cognitivo como tarea de la educación. En M. Arca, P. Guidoni y P. Mazzoli, Enseñar Ciencia. Como empezar: Reflexiones para una educación científica. Barcelona: Editorial Paidós.

Archila, P. (2013). La argumentación y sus aportes a la enseñanza bilingüe. Revista Eureka sobre Enseñanza y Divulgación de las Ciencias, 10(3), 406-423. https://doi.org/10.25267/Rev_Eureka_ensen_divulg_ cienc.2013.v10.i3.07

Bahamón, X. (2009). La clase de ciencias como espacio experiencial para el despliegue de habilidades comunicativas. Bogotá D.C.: Universidad Pedagógica Nacional.

Barrio, I. et al. (2012). Métodos de Investigación Educativa: el estudio de caso. Madrid: Universidad Autónoma de Madrid.

Buck, G. (2000). Teaching Science to English-asSecond-Language Learners: Teaching, learning, and assessment strategies for elementary ESL students. Faculty Publications: Department of Teaching, Learning and Teacher Education. Recuperado de http://digitalcommons.unl.edu/cgi/viewcontent. cgi?article $=1018 E$ context $=$ teachlearnfacpub

Candela, A. (1999). Discurso de la ciencia en el aula. En A. Pellicer, A. Candela, J. Kalman, E. Taboada, R. Quiroz, E. Remedi, D. Block, Encuentro de Investigación Educativa 95-98 (pp. 35-48). México: Editorial Plaza y Valdés.

Choi, J. y Kuipers, J. (2003). Bilingual Practices in a Science Classroom: Bilingual Hispanic Students' Ways of Constructing School Science. USA: National Science Foundation; U.S. Department of Education \& National Institute of Health. Recuperado de https://www2.gwu.edu/ scale-up/ documents/EQRE.pdf 
Coyle, D. Hood, P. y Marsh, D. (2010). Content and language integrated learning. Cambridge: Cambridge University Press.

De Mejía, A. (1998). Educación bilingüe en Colombia en contextos lingüísticos mayoritarios: hacia una caracterización del campo. Bilingüismo. Función Cognitiva y Educación, 26, 8-23.

De Mejía, A., Ordóñez, C. y Fonseca, L. (2006). Colombia Aprende. Lineamientos para la Educación Bilingüe en Colombia: Hacia una política coherente - Estudio Investigativo sobre el estado actual de la educación bilingüe (Inglés-Español) en Colombia. Bogotá: Centro de Investigación y Formación en Educación. Universidad de los Andes. Recuperado de http:// www.colombiaaprende.edu.co/html/productos/1685/ articles-266111_archivo_1.pdf

Elkana, Y. (1983). La ciencia como sistema cultural (Una aproximación antropológica). Boletín de la Sociedad Colombiana de Epistemología, 3 (10-11).

García, S. (2009). La enseñanza bilingüe en las ciencias experimentales. Revista Digital Innovación $y$ Experiencias, 1-9.

Geertz, C. (1973). La interpretación de las culturas. Barcelona: Editorial Gedisa, S.A.

Jaipal, K. (2011). A semiotic discurse analyses framework: Understanding meaning making in science education contexts. En K. Jaipal, Semiotic Theory an Applications (pp. 191-208). St. Catharines: Editorial Nova Science Publishers, Inc.

Jarrett, D. (1999). The inclusive classroom: Teaching mathematics and science to english- language learners. Northwest Regional Educational Laboratory. Recuperado de https://eric.ed.gov/?id=ED455690

Lemke, J. (1997). Apreder a hablar ciencia. Barcelona: Editorial Paidós.

Lotman, Y. (1996). Acerca de la Semiosfera. En Y. Lotman, La Semiosfera I: semiótica de la cultura del texto (p. 21-42). Madrid: Ediciones Cátedra.
Mariño, C. (2014). Towards implementing CLIL (Content and Language Integrated Learning) at CBS (Tunja, Colombia). Colombian Applied Linguistics Journal, 16(2), 151-160. https://doi.org/10.14483/udistrital. jour.calj.2014.2.a02

Ministerio de Educación Nacional. (2005). Bases para una nación bilingüe y competitiva. Altablero, 37 (Octubre-Diciembre).

Meyer, O. (2010). Towards quality-CLIL: successful planning and teaching strategies. Plus, 33, 11-29.

Rincón, M. (2017). El origen del concepto de Ecosistemas. Revista Bio-grafía. Escritos sobre biología y sus enseñanzas. (pp. 342-350). https:// doi.org/10.17227/20271034.vol.0num.0biografia342.350

Secretaría de Educación Distrital (SED). (2007). Colegios Públicos de excelencia para Bogotá - Orientaciones curriculares para el campo de Ciencia y Tecnología. Recuperado de https://www.educacionbogota. edu.co/Centro Documentacion/anexos/ publicaciones_2004̄_2008/101083_Ciecia\%20y\%20 tecnologia_bja.pdf

Snow, C. (1999). Bilingüismo y adquisición de una segunda lengua. En J. Berko y N. Berstain, Psicolingüústica (pp. 477- 507). Madrid: McGraw Hill.

Suárez, J. (2013). Construcción de una propuesta de enseñanza de las ciencias naturales en segunda lengua. Bogotá: Universidad Pedagógica Nacional.

Zamora, G. y Zerón, A. (2010). Caracterización y sentido actual de la autoridad pedagógica en escuelas chilenas de sectores de pobreza. Revista Española de Pedagogía (REP), 68(245), 99-115.

Zydatib, W. (2007). Bilungualer fachunterricht in Deutchsland: einebilanz. Fremdspraschen Lehren und Lernen, 36, 8-25. 